\title{
European Antibiotic Awareness Day: a five-year perspective of Europe-wide actions to promote prudent use of antibiotics
}

S Earnshaw ${ }^{1,2}$, G Mancarella (giovanni.mancarella@ecdc.europa.eu) ${ }^{1,2}$, A Mendez $^{1}$, B Todorova ${ }^{1}$, A P Magiorakos ${ }^{1}$, E Possenti ${ }^{1}$, M $^{2}$ Stryk $^{1}$, S Gilbro ${ }^{1}$, H Goossens ${ }^{3,4,5}$, B Albiger ${ }^{1}$, D L Monnet ${ }^{1}$, on behalf of the European Antibiotic Awareness Day Technical Advisory Committee $^{6}$, on behalf of the European Antibiotic Awareness Day Collaborative Group ${ }^{7}$

1. European Centre for Disease Prevention and Control, Stockholm, Sweden

2. These authors contributed equally to this work

3. Laboratory of Medical Microbiology, Vaccine and Infectious Disease Institute, University of Antwerp, Antwerp, Belgium

4. European Antibiotic Awareness Day Technical Advisory Committee

5. European Antibiotic Awareness Day Collaborative Group

6. Members of the Technical Advisory Committee are listed at the end of the article

7. Members of the Collaborative Group are listed at the end of the article

Citation style for this article:

Earnshaw S, Mancarella G, Mendez A, Todorova B, Magiorakos AP, Possenti E, Stryk M, Gilbro S, Goossens H, Albiger B, Monnet DL, on behalf of the European

Antibiotic Awareness Day Technical Advisory Committee, on behalf of the European Antibiotic Awareness Day Collaborative Group. European Antibiotic Awareness Day: a five-year perspective of Europe-wide actions to promote prudent use of antibiotics. Euro Surveill. 2014;19(41):pii=20928. Available online: http://www. eurosurveillance.org/ViewArticle.aspx?Articleld=20928

Article submitted on 31 October 2013 / published on 16 October 2014

Following the European Union (EU) Council Recommendation on prudent use of antimicrobial agents in human medicine in 2001, and the success of national campaigns, i.e. Belgium and France, the European Centre for Disease Prevention and Control (ECDC) decided to establish the European Antibiotic Awareness Day (EAAD) on 18 November as platform to support national campaigns across Europe. This article provides an overview of EAAD tools, materials, and activities developed during the first five years. It shows that EAAD has been successful due to good cooperation between ECDC and national institutions, strong political and stakeholder support and evidencebased development of campaign materials. EAAD has provided a platform for pre-existing national campaigns and encouraged similar campaigns to develop where neither political support had been secured, nor financial support had been available. As a result, participating countries have continuously expressed strong support for ECDC to continue its work on EAAD. This has been endorsed by a steadily increasing number of countries participating and the growing interest of varied professional and stakeholder organisations. We conclude that EAAD should continue to act as catalyst for discussion and as mechanism to raise awareness of the public and prescribers about prudent use of antibiotics.

\section{Introduction}

The emergence and spread of antibiotic resistance, is recognised as a global problem. Its immediate consequence is that, only a limited number of antibiotics, and sometimes even no antibiotic, is available for the treatment of infections caused by resistant bacteria. Other direct consequences for patients include delayed administration of appropriate antibiotic therapy, longer stays in hospitals, higher healthcare costs and poor patient outcomes [1]. Worldwide action is thus necessary to avert an impending threat to human health [2].

Of the steps that need to be taken to address antibiotic resistance, we believe that improving antibiotic use is the most important action needed to greatly slow the development and spread of antibiotic-resistant bacteria. Antibiotics are frequently used inappropriately or when they are not needed, in both humans and animals.

Following adoption of the European Union (EU) Council Recommendation on the prudent use of antimicrobial agents in human medicine in November 2001, which stated that EU Member States should inform the general public of the importance of prudent use of antimicrobial agents and the success of some national campaigns, such as Belgium and France, the European Centre for Disease Prevention and Control (ECDC) decided in 2008 to establish the European Antibiotic Awareness Day (EAAD) on 18 November as a platform for providing support to national campaigns across the region [3].

Since 2008, numerous health-related and professional organisations, as well as the European Commission and the World Health Organization Regional Office for Europe (WHO/Europe), have partnered with ECDC in preparing communications materials and planning activities targeting both communities and hospitals for EAAD. In 2012, under the banner of EAAD, national campaigns to inform about prudent antibiotic use took place in 43 European countries, with the target audiences selected by campaign organisers at national level, including both general public and prescribers. 
This perspective describes the development of materials and tools during the past five years, and provides a review of the activities and achievements of EAAD. It also presents results from the annual questionnaire provided by participating countries and from an independent monitoring of the media coverage.

\section{Development of materials and tools for the campaigns}

ECDC endeavoured throughout the year 2008 to provide participating countries with a core set of tools, including a common name 'European Antibiotic Awareness Day' and logo, key messages, a dedicated website and communications materials targeting parents and carers of young children $[4,5]$. The various steps in preparation for the first EAAD that took place on 18 November 2008 were previously published [6].

In the following years, EAAD has focussed on primary care prescribers (2009) and hospital prescribers (2010). In each case, campaign messages and materials were developed following evidence-based processes, i.e. results of systematic reviews and subsequently they were reviewed by the EAAD Technical Advisory Committee and tested in focus groups representing the target audience in question. The campaign materials included a dedicated EAAD website, logos and visuals i.e., hedgehog mascot and TV and web spots, advertorials and on line banners, factsheets and prescribing check lists, patient brochures, template letters and presentations. All campaign materials were made available on the EAAD website [7].

In 2011 and 2012, the focus of EAAD shifted to consolidation, with new activities to support the national campaigns at a process level as opposed to the development of new content $[8,9]$. Given the global financial crisis and competing priorities, a number of countries reviewed government support for the annual campaigns. ECDC chose to strengthen its support to the participating countries by providing strategies and tools to support the delivery of the existing key messages and materials including a social media toolkit, and to foster impact evaluation strategies, and a pilot training course on development, implementation and evaluation of prudent antibiotic use campaigns.

Each year, participating countries answered a questionnaire providing feedback to ECDC on their national activities for EAAD. The scope of this questionnaire is to gather information about the national campaigns: e.g. type and number of the activities, chosen target audience governmental support and EAAD material used to support those activities. In addition since 2010, an independent monitoring of the media coverage of EAAD in terms of print, online and social media is performed.

\section{Coverage of the campaigns}

Since 2008, the number of European countries participating in the EAAD has increased year on year. In 2008, all EU Member States plus Norway, Iceland, Croatia,

\section{FIGURE}

Countries participating in the European Antibiotic Awareness Day, 2008-2012

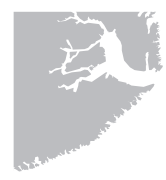

Participating countries since:

$2008(n=32)$

$2009(n=33)$

$2010(n=35)$

$2011(n=37)$

$2012(n=43)$

Non-participating countries

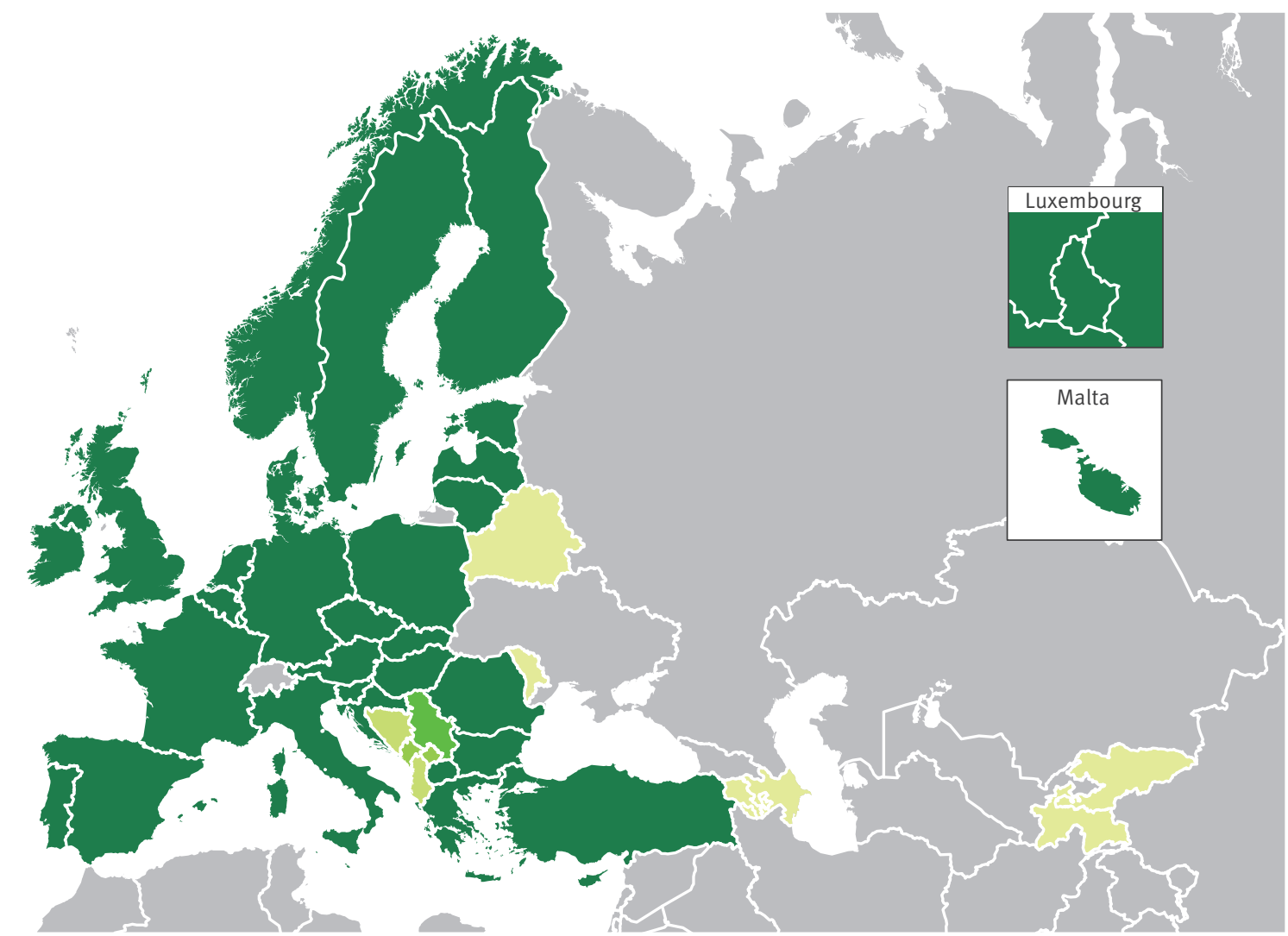


the Former Yugoslav Republic of Macedonia and Turkey participated making a total of 32 countries. Between 2009 and 2012, this number increased to 43 countries, firstly with the addition of other EU enlargement countries: Albania, Bosnia and Herzegovina, Kosovo*, Montenegro and Serbia [10,11]. In 2012, through cooperation with WHO/Europe, Armenia, Azerbaijan, Belarus, Kyrgyzstan, Moldavia and Tajikistan also participated (Figure).

Thirty-two countries initially participated to EAAD in 2008; one additional country in 2009, two additional countries in 2010; in 2011 two further countries joined and six more in 2012. Thus in 2012, a total of 43 European countries participated and in 2013, the number countries reached 45 (unpublished data).

Each participating country has carried out at least one activity targeting the general public, primary care prescribers or hospital prescribers (Table 1). The target audiences have predominantly followed the theme set by ECDC at European level, i.e. twenty-seven of 33 participating countries targeted primary care prescribers in 2009 and 31 of 35 countries targeted hospital prescribers in 2010 [12]. In the subsequent years of consolidation, ECDC has seen a continued focus on all three target audiences with in 2012, 36 of 43 countries organising activities targeted at the general public, 34 at primary care prescribers and 30 at hospital prescribers (Table 1).

\section{Governmental support}

Government support has been an essential element in funding and endorsing national campaigns. This support was universal in 2008 , but then probably due to financial constraints and/or competing priorities (e.g. the 2009 influenza $A\left(\mathrm{H}_{1} \mathrm{~N}_{1}\right)$ pdm pandemic), a number of countries were not able to secure on-going government support for the annual campaigns. In 2009, 23 countries had government support, of which 14 were able to secure funding for their national campaigns as part of this support. The level of government funding further decreased and in 2010 only nine countries remained with government funding. This number increased again by 2012 , with 30 countries then receiving support, of which 15 received financial support, from their governments (Table 2).

As government support varied in 2009 and 2010, campaign planners considered a broader scope of alternative groups to provide support and funding, such as professional groups and non-governmental organisations. By 2010, 16 countries reported cooperation with professional groups, such as medical associations and professional healthcare organisations. In 2011 and 2012, this number increased to 27 and 35 countries, respectively, of which 10 countries and 19 countries, respectively, reported receiving sponsorships (Table 2).
Print, online and social media coverage

ECDC has consistently monitored print and online press coverage of EAAD in all 24 official EU languages since 2010. In 2010 and in 2011, 476 and 611 articles related to EAAD were published, respectively, during a fourmonth period between 15 October and 15 February. In 2012, 446 articles related to EAAD were published in 47 countries worldwide during a two-month monitoring period between 18 October and 28 December. This coverage represented a range of 42 to 72 million visits of news online and a print reach of 18 to 77 million persons.

The EAAD website (http://ecdc.europa.eu/en/EAAD/ Pages/Home.aspx) includes communications materials in all EU languages [7]. Analysis of the EAAD website showed around a $200 \%$ increase in web traffic i.e., during the week of 18 November each year compared to the previous one. The most visited EAAD pages were the country activities, toolkits, multimedia news release (for English version), as well as the factsheets and national campaigns (for the multilingual websites).

Since 2011, ECDC has increasingly used social media (e.g. Twitter, Facebook) to convey EAAD messages. In 2012, EAAD was mentioned in 1,773 tweets, with over of 3.7 million impressions reached. In 2012, ECDC with WHO/Europe and the European Commission held a joint Twitter chat on 20 November reaching 2.5 million impressions (out of the 3.7 million stated above). EAAD was also mentioned 58-times in the monitored period in blogs, e.g. European Medical Students' Association. The postings focused on the EAAD and the use of antibiotics.

From 2009 to 2012, ECDC broadcasted a TV spot raising awareness on antimicrobial resistance and EAAD on a pan-European TV channel (Euronews), reaching an average of 14 million EU citizens each year and among them an average of 1.5 million people working in the healthcare and medicine sector in Europe.

\section{Discussion}

In 2007, when the idea of a European-level initiative to raise awareness about the importance of prudent use of antibiotics was agreed, ECDC hosted two meetings of national antimicrobial resistance (AMR) focal points, nominated by the Member States. In these meetings in September 2007 and March 2008, the form that the initiative should take and the benefits that it could bring were discussed as well as draft campaign materials, and feedback was given. In the end, the initiative was conceived as a day (EAAD) upon which national campaigns could be launched and where the power of many could amount to more than the power of one $[6,13-16]$. Our analysis after five editions of EAAD, shows that it obviously responded to a need at European level.

The EAAD has provided a platform for pre-existing national campaigns and encouraged similar campaigns to develop in other countries where neither political support had been secured, nor financial support been 


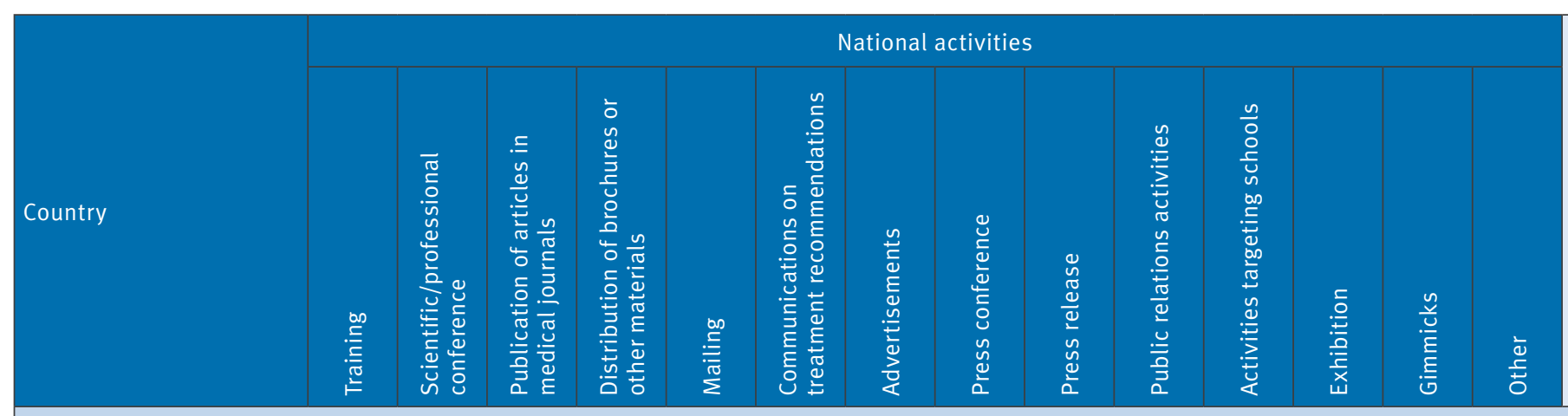

European Union

\begin{tabular}{|c|c|c|c|c|c|c|c|c|c|c|c|c|c|c|}
\hline Austria & & $\bullet \bullet$ & $\bullet \bullet$ & $\bullet$ & & & & $\bullet \bullet$ & $\bullet \bullet$ & $\bullet$ & & & & $\bullet \bullet$ \\
\hline Belgium & & $\bullet$ & $\bullet \bullet$ & $\bullet$ & $\bullet$ & $\bullet \bullet$ & & $\bullet \bullet$ & $\bullet \bullet \bullet$ & & $\bullet$ & $\bullet$ & $\bullet$ & $\bullet$ \\
\hline Bulgaria & $\bullet \bullet$ & $\bullet \bullet \bullet$ & $\bullet \bullet$ & $\bullet$ & $\bullet \bullet \bullet$ & $\bullet$ & & $\bullet \bullet \bullet$ & $\bullet \bullet$ & $\bullet$ & & $\bullet$ & & \\
\hline Croatia & & $\bullet \bullet \bullet$ & $\bullet \bullet$ & $\bullet \bullet \bullet$ & $\bullet$ & $\bullet$ & & $\bullet \bullet \bullet$ & $\bullet$ & $\bullet \bullet$ & $\bullet$ & & $\bullet$ & $\bullet \bullet$ \\
\hline Cyprus & $\bullet \bullet$ & $\bullet \bullet$ & & $\bullet \bullet$ & $\bullet$ & $\bullet$ & $\bullet$ & $\bullet$ & $\bullet \bullet \bullet$ & $\bullet \bullet$ & & $\bullet$ & & $\bullet \bullet$ \\
\hline Czech Republic & $\bullet$ & $\bullet \bullet$ & $\bullet \bullet$ & $\bullet \bullet$ & $\bullet \bullet$ & $\bullet$ & & $\bullet$ & $\bullet \bullet$ & $\bullet$ & & $\bullet$ & $\bullet$ & $\bullet \bullet$ \\
\hline Denmark & & $\bullet \bullet \bullet$ & $\bullet \bullet$ & & & $\bullet$ & & & $\bullet \bullet$ & & & & & $\bullet \bullet$ \\
\hline Estonia & & $\bullet$ & $\bullet \bullet \bullet$ & $\bullet$ & $\bullet \bullet \bullet$ & $\bullet$ & $\bullet$ & $\bullet \bullet$ & $\bullet \bullet \bullet$ & $\bullet$ & $\bullet$ & & & \\
\hline Finland & & & & & & & & $\bullet$ & $\bullet \bullet$ & & & & & $\bullet \bullet$ \\
\hline France & $\bullet$ & $\bullet$ & $\bullet$ & $\bullet$ & & $\bullet$ & & & $\bullet$ & $\bullet$ & & & & $\bullet \bullet$ \\
\hline Germany & $\bullet$ & $\bullet \bullet \bullet$ & $\bullet \bullet \bullet$ & & & & & & $\bullet \bullet$ & & & & & $\bullet \bullet$ \\
\hline Greece & $\bullet \bullet$ & $\bullet$ & $\bullet \bullet$ & $\bullet$ & & $\bullet$ & & $\bullet \bullet$ & $\bullet \bullet$ & $\bullet \bullet$ & & & & $\bullet \bullet$ \\
\hline Hungary & & $\bullet \bullet \bullet$ & $\bullet \bullet$ & $\bullet \bullet$ & $\bullet$ & & $\bullet$ & $\bullet \bullet \bullet$ & $\bullet \bullet \bullet$ & $\bullet \bullet$ & & & & \\
\hline Ireland & & $\bullet$ & $\bullet$ & $\bullet \bullet$ & $\bullet \bullet$ & $\bullet$ & $\bullet$ & $\bullet \bullet \bullet$ & $\bullet \bullet$ & $\bullet \bullet$ & & & $\bullet$ & $\bullet$ \\
\hline Italy & & $\bullet \bullet$ & & $\bullet$ & & & & $\bullet$ & $\bullet \bullet$ & $\bullet \bullet$ & $\bullet \bullet$ & & & $\bullet \bullet \bullet$ \\
\hline Latvia & - & $\bullet \bullet$ & $\bullet \bullet \bullet$ & & $\bullet$ & & & $\bullet$ & $\bullet$ & $\bullet$ & & & & \\
\hline Lithuania & $\bullet$ & $\bullet \bullet$ & $\bullet \bullet$ & $\bullet$ & $\bullet$ & & $\bullet$ & & $\bullet \bullet$ & $\bullet$ & $\bullet \bullet$ & & & $\bullet \bullet$ \\
\hline Luxembourg & & $\bullet$ & $\bullet$ & $\bullet$ & $\bullet \bullet$ & $\bullet$ & $\bullet$ & $\bullet$ & $\bullet \bullet$ & $\bullet$ & & & & $\bullet$ \\
\hline Malta & & $\bullet \bullet$ & $\bullet$ & $\bullet$ & $\bullet$ & & $\bullet$ & $\bullet$ & $\bullet$ & $\bullet$ & & & & $\bullet \bullet$ \\
\hline Netherlands & & & $\bullet$ & $\bullet$ & $\bullet$ & & & $\bullet$ & $\bullet \bullet$ & & & & & $\bullet$ \\
\hline Poland & $\bullet \bullet \bullet$ & $\bullet \bullet \bullet$ & $\bullet \bullet \bullet \bullet$ & $\bullet \bullet \bullet \bullet$ & $\bullet$ & $\bullet \bullet \bullet$ & $\bullet \bullet$ & $\bullet \bullet$ & $\bullet \bullet \bullet$ & $\bullet \bullet$ & $\bullet \bullet$ & $\bullet \bullet \bullet$ & & $\bullet \bullet$ \\
\hline Portugal & & $\bullet \bullet$ & $\bullet$ & $\bullet$ & & $\bullet$ & $\bullet$ & $\bullet$ & $\bullet \bullet$ & $\bullet \bullet$ & $\bullet \bullet$ & $\bullet$ & & $\bullet$ \\
\hline Romania & $\bullet$ & $\bullet \bullet$ & $\bullet \bullet$ & $\bullet$ & $\bullet \bullet \bullet$ & $\bullet \bullet$ & & $\bullet \bullet$ & $\bullet \bullet$ & $\bullet \bullet$ & & & & $\bullet$ \\
\hline Slovakia & $\bullet$ & $\bullet \bullet$ & $\bullet$ & $\bullet$ & $\bullet$ & $\bullet$ & & $\bullet \bullet$ & $\bullet \bullet$ & $\bullet$ & $\bullet$ & & & \\
\hline Slovenia & $\bullet$ & $\bullet \bullet$ & $\bullet \bullet \bullet$ & & $\bullet$ & & & $\bullet$ & $\bullet \bullet$ & $\bullet$ & & & & $\bullet \bullet$ \\
\hline Spain & & $\bullet \bullet \bullet$ & $\bullet \bullet \bullet$ & $\bullet \bullet \bullet$ & $\bullet \bullet \bullet$ & $\bullet$ & $\bullet \bullet \bullet$ & $\bullet \bullet$ & $\bullet \bullet \bullet$ & $\bullet \bullet$ & $\bullet \bullet$ & $\bullet \bullet$ & & $\bullet$ \\
\hline Sweden & & $\bullet$ & $\bullet \bullet$ & $\bullet$ & $\bullet$ & & & $\bullet$ & $\bullet \bullet$ & & & & & $\bullet \bullet$ \\
\hline United Kingdom & $\bullet$ & $\bullet \bullet \bullet$ & $\bullet \bullet \bullet$ & $\bullet \bullet$ & $\bullet \bullet$ & $\bullet$ & & & $\bullet \bullet$ & $\bullet$ & $\bullet \bullet$ & $\bullet$ & & $\bullet \bullet \bullet$ \\
\hline
\end{tabular}

European Economic Area

Iceland

Norway

\begin{tabular}{|l|l|l|l|l|l|}
\hline & $\bullet$ & & $\bullet$ & $\bullet$ & $\bullet$ \\
\hline & $\bullet \bullet$ & $\bullet \bullet$ & $\bullet \bullet$ & $\bullet$ & $\bullet$ \\
\hline
\end{tabular}

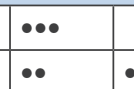

Other countries

\begin{tabular}{|c|c|c|c|c|c|c|c|c|c|c|c|c|c|}
\hline Albania & & $\bullet \bullet$ & $\bullet$ & $\bullet$ & & $\bullet \bullet$ & $\bullet$ & $\bullet$ & - & $\bullet \bullet$ & & - & \\
\hline Bosnia and Herzegovina & $\bullet$ & $\bullet$ & $\bullet$ & $\bullet \bullet$ & $\bullet$ & $\bullet$ & $\bullet$ & $\bullet$ & $\bullet$ & $\bullet$ & & & \\
\hline $\begin{array}{l}\text { Former Yugoslav Republic } \\
\text { of Macedonia }\end{array}$ & & $\bullet \bullet \bullet$ & $\bullet \bullet \bullet$ & $\bullet \bullet$ & & & - & $\bullet \bullet \bullet$ & $\bullet \bullet$ & $\bullet \bullet$ & & & $\bullet$ \\
\hline Kosovo* & $\bullet$ & $\bullet \bullet$ & & $\bullet \bullet \bullet$ & $\bullet \bullet \bullet$ & & & $\bullet \bullet$ & $\bullet \bullet$ & $\bullet \bullet$ & & & $\bullet$ \\
\hline Montenegro & & $\bullet$ & & & & & & & & $\bullet$ & & & $\bullet$ \\
\hline Serbia & $\bullet \bullet$ & $\bullet$ & $\bullet$ & & $\bullet \bullet$ & $\bullet$ & & $\bullet \bullet \bullet$ & $\bullet \bullet$ & $\bullet \bullet$ & & & $\bullet \bullet$ \\
\hline Turkey & $\bullet$ & $\bullet \bullet \bullet$ & & $\bullet \bullet \bullet$ & $\bullet \bullet$ & $\bullet$ & & $\bullet \bullet \bullet$ & $\bullet \bullet$ & $\bullet$ & $\bullet \bullet$ & & $\bullet \bullet$ \\
\hline
\end{tabular}

Each dot corresponds to one activity-year.

* This designation is without prejudice to positions on status, and is in line with United Nations Security Council Resolution 1244/99 and the International Court of Justice Opinion on the Kosovo declaration of independence. 


\begin{tabular}{|c|c|c|c|c|c|c|c|c|c|c|c|c|c|c|c|}
\hline & - & & & & & & • & - & & $\bullet$ & • & & & & $\bullet$ \\
\hline & & & & $\bullet$ & & & $\bullet \bullet \bullet \bullet$ & $\bullet \bullet \bullet$ & & $\bullet \bullet \bullet$ & $\bullet \bullet \bullet \bullet$ & & $\bullet \bullet \bullet$ & & - \\
\hline$\bullet \bullet$ & $\bullet$ & & $\bullet$ & $\bullet$ & & & $\bullet \bullet$ & $\bullet \bullet$ & $\bullet$ & $\bullet \bullet \bullet$ & $\bullet \bullet \bullet$ & - & & & \\
\hline$\bullet \bullet$ & $\bullet \bullet$ & & & $\bullet$ & & & $\bullet \bullet \bullet$ & $\bullet \bullet \bullet$ & & $\bullet \bullet \bullet$ & $\bullet$ & & $\bullet \bullet$ & $\bullet$ & - \\
\hline$\bullet \bullet$ & & & $\bullet$ & $\bullet$ & & & $\bullet \bullet$ & $\bullet \bullet \bullet$ & $\bullet$ & $\bullet$ & $\bullet \bullet$ & $\bullet \bullet$ & $\bullet$ & - & $\bullet$ \\
\hline \multirow[t]{2}{*}{$\bullet \bullet$} & $\bullet \bullet$ & & $\bullet \bullet$ & • & $\bullet \bullet$ & • & $\bullet \bullet \bullet$ & $\bullet \bullet \bullet$ & & • & $\bullet \bullet \bullet$ & $\bullet \bullet$ & $\bullet$ & $\bullet$ & $\bullet$ \\
\hline & & & & $\bullet$ & & & $\bullet$ & $\bullet$ & & $\bullet$ & $\bullet \bullet$ & & & & $\bullet$ \\
\hline • & & & & & & & & $\bullet$ & & $\bullet \bullet \bullet$ & $\bullet \bullet \bullet$ & & $\bullet$ & & • \\
\hline \multirow[t]{3}{*}{ • } & & & & & & & & & & & $\bullet \bullet$ & & & & • \\
\hline & $\bullet$ & - & & $\bullet$ & & & & $\bullet$ & & $\bullet$ & $\bullet$ & & $\bullet$ & & $\bullet \bullet$ \\
\hline & & & & $\bullet$ & & & & & & & $\bullet$ & & & & $\bullet \bullet \bullet$ \\
\hline - & & $\bullet$ & & $\bullet$ & & & $\bullet \bullet$ & $\bullet \bullet$ & & & $\bullet$ & - & 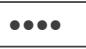 & - & $\bullet$ \\
\hline$\bullet \bullet \bullet$ & - & & & $\bullet \bullet$ & $\bullet$ & & $\bullet \bullet \bullet$ & $\bullet \bullet \bullet$ & - & $\bullet \bullet$ & $\bullet \bullet \bullet$ & - & $\bullet \bullet$ & & \\
\hline$\bullet \bullet$ & $\bullet$ & & & $\bullet \bullet \bullet$ & - & & $\bullet \bullet$ & $\bullet \bullet$ & & $\bullet \bullet$ & $\bullet \bullet$ & $\bullet$ & $\bullet$ & $\bullet$ & $\bullet \bullet \bullet$ \\
\hline$\bullet$ & & & & & & & $\bullet$ & & & & $\bullet \bullet \bullet$ & $\bullet$ & $\bullet$ & $\bullet$ & $\bullet$ \\
\hline - & & & & $\bullet$ & & & & & & • & $\bullet \bullet \bullet$ & & $\bullet \bullet$ & & \\
\hline$\bullet \bullet$ & & & $\bullet$ & $\bullet$ & & & $\bullet \bullet \bullet$ & $\bullet \bullet$ & & $\bullet$ & $\bullet \bullet \bullet$ & $\bullet$ & $\bullet$ & $\bullet$ & $\bullet \bullet$ \\
\hline$\bullet$ & & & & & & & $\bullet$ & $\bullet$ & & $\bullet \bullet$ & $\bullet$ & $\bullet$ & $\bullet$ & - & $\bullet$ \\
\hline - & - & & & & & & $\bullet \bullet$ & $\bullet \bullet \bullet$ & & $\bullet$ & $\bullet$ & $\bullet \bullet$ & $\bullet \bullet$ & & $\bullet \bullet$ \\
\hline$\bullet$ & $\bullet$ & & & & & & $\bullet$ & $\bullet$ & - & $\bullet \bullet$ & $\bullet \bullet$ & $\bullet$ & & & \\
\hline$\bullet \bullet \bullet$ & $\bullet \bullet \bullet$ & - & & $\bullet$ & $\bullet \bullet \bullet$ & & 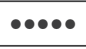 & 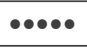 & & $\bullet$ & 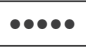 & $\bullet$ & 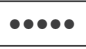 & $\bullet$ & $\bullet$ \\
\hline$\bullet \bullet$ & & & & - & & & $\bullet \bullet$ & $\bullet \bullet$ & - & $\bullet$ & 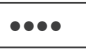 & $\bullet \bullet$ & $\bullet \bullet \bullet$ & $\bullet$ & • \\
\hline \multirow[t]{2}{*}{ 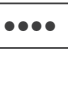 } & $\bullet \bullet \bullet$ & $\bullet \bullet$ & $\bullet \bullet$ & $\bullet$ & & & $\bullet$ & $\bullet \bullet \bullet \bullet$ & $\bullet \bullet$ & $\bullet \bullet$ & $\bullet \bullet \bullet \bullet$ & $\bullet$ & $\bullet$ & $\bullet \bullet \bullet$ & $\bullet$ \\
\hline & & & & $\bullet$ & & & - & $\bullet$ & & $\bullet$ & $\bullet$ & & $\bullet$ & $\bullet$ & $\bullet$ \\
\hline$\bullet$ & & & & $\bullet$ & & & $\bullet \bullet$ & $\bullet$ & & $\bullet \bullet$ & $\bullet$ & & $\bullet$ & & $\bullet$ \\
\hline$\bullet \bullet$ & $\bullet \bullet \bullet$ & $\bullet \bullet$ & $\bullet \bullet$ & $\bullet \bullet \bullet$ & - & & 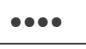 & $\bullet \bullet \bullet$ & $\bullet$ & 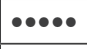 & $\bullet \bullet \bullet$ & $\bullet \bullet$ & $\bullet$ & & $\bullet$ \\
\hline$\bullet$ & & & & $\bullet$ & & & $\bullet$ & & & $\bullet \bullet$ & & $\bullet \bullet$ & $\bullet$ & & $\bullet$ \\
\hline$\bullet \bullet$ & $\bullet \bullet$ & $\bullet \bullet$ & $\bullet \bullet$ & $\bullet$ & $\bullet$ & & $\bullet \bullet \bullet$ & $\bullet \bullet \bullet$ & - & $\bullet \bullet \bullet$ & $\bullet \bullet \bullet$ & $\bullet$ & $\bullet$ & - & $\bullet \bullet$ \\
\hline$\bullet$ & & & & - & & & & & & & $\bullet \bullet \bullet$ & - & & & - \\
\hline$\bullet$ & & & & - & & & $\bullet$ & $\bullet \bullet$ & & $\bullet \bullet$ & $\bullet$ & $\bullet$ & $\bullet$ & $\bullet$ & $\bullet$ \\
\hline$\bullet \bullet$ & & & & & & & $\bullet \bullet$ & $\bullet \bullet$ & & $\bullet \bullet$ & & & $\bullet$ & & \\
\hline$\bullet$ & & & & $\bullet \bullet$ & & & $\bullet$ & $\bullet$ & & $\bullet$ & $\bullet$ & $\bullet$ & $\bullet$ & $\bullet$ & \\
\hline$\bullet \bullet$ & $\bullet$ & & & $\bullet$ & & & $\bullet \bullet$ & $\bullet$ & $\bullet$ & $\bullet \bullet$ & $\bullet$ & $\bullet$ & $\bullet$ & • & $\bullet$ \\
\hline$\bullet \bullet \bullet$ & $\bullet$ & & & $\bullet \bullet \bullet$ & & & $\bullet$ & $\bullet \bullet$ & - & $\bullet \bullet \bullet$ & & - & & & \\
\hline & & & & $\bullet$ & & & $\bullet$ & $\bullet$ & & $\bullet$ & & & & & \\
\hline & & & & $\bullet$ & & & $\bullet$ & & & $\bullet \bullet$ & $\bullet \bullet$ & & & & \\
\hline$\bullet \bullet \bullet$ & $\bullet \bullet$ & $\bullet \bullet$ & $\bullet$ & $\bullet \bullet$ & $\bullet \bullet$ & & $\bullet \bullet$ & & $\bullet \bullet$ & $\bullet \bullet \bullet$ & $\bullet \bullet$ & & $\bullet \bullet \bullet$ & & $\bullet \bullet$ \\
\hline
\end{tabular}




\section{TABLE 2}

Overview of government and stakeholders' support received per country, European Antibiotic Awareness Day campaigns, $2008-2012$

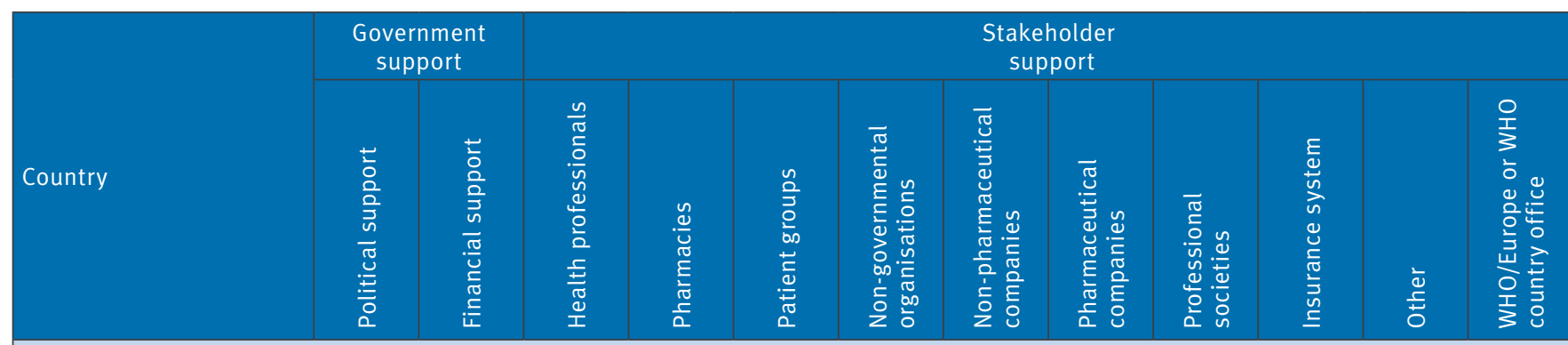

\section{European Union}

\begin{tabular}{|c|c|c|c|c|c|c|c|c|c|c|c|c|}
\hline Austria & $\bullet \bullet \bullet$ & $\bullet$ & $\bullet \bullet$ & $\bullet$ & & $\bullet$ & $\bullet \bullet$ & $\bullet$ & $\bullet$ & $\bullet$ & $\bullet$ & \\
\hline Belgium & $\bullet \bullet \bullet$ & 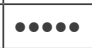 & $\bullet$ & $\bullet \bullet \bullet \bullet$ & & & $\bullet$ & $\bullet$ & $\bullet \bullet \bullet$ & $\bullet \bullet \bullet$ & & \\
\hline Bulgaria & $\bullet$ & & $\bullet$ & $\bullet$ & & $\bullet \bullet$ & - & $\bullet$ & $\bullet \bullet$ & & & $\bullet$ \\
\hline Croatia & $\bullet$ & $\bullet \bullet \bullet \bullet$ & $\bullet \bullet \bullet \bullet$ & $\cdots \bullet \bullet$ & & $\bullet \bullet \bullet$ & & $\cdot$ & $\bullet \bullet$ & & & $\bullet$ \\
\hline Cyprus & $\bullet \bullet \bullet$ & $\bullet \bullet$ & $\bullet$ & $\bullet$ & & & $\bullet$ & $\cdot$ & & $\bullet$ & & \\
\hline Czech Republic & $\bullet \bullet \bullet$ & & $\bullet$ & $\bullet \bullet \bullet$ & $\bullet$ & & $\bullet$ & & $\bullet \bullet \bullet$ & $\bullet$ & $\bullet$ & $\bullet$ \\
\hline Denmark & $\bullet$ & $\bullet$ & $\bullet$ & $\bullet$ & & & & & $\cdot$ & & $\bullet$ & \\
\hline Estonia & $\bullet$ & & $\bullet$ & & & & & & $\bullet$ & & & \\
\hline Finland & $\bullet$ & $\bullet$ & $\bullet$ & & & & & & & & & \\
\hline France & $\bullet \bullet \bullet$ & $\bullet \bullet$ & $\bullet$ & & & & & & $\bullet \bullet$ & $\bullet \bullet$ & $\bullet$ & \\
\hline Germany & $\bullet \bullet \bullet$ & $\bullet \bullet$ & $\bullet$ & & & & & & $\bullet \bullet$ & & $\bullet$ & \\
\hline Greece & $\bullet$ & $\bullet$ & $\bullet$ & & & & & $\bullet$ & $\bullet \bullet \bullet$ & & $\bullet$ & \\
\hline Hungary & $\bullet \bullet \bullet$ & $\bullet$ & & & & & & & & & & • \\
\hline Ireland & $\bullet$ & $\bullet$ & $\bullet \bullet \bullet$ & $\bullet$ & $\bullet$ & & & & $\bullet \bullet$ & & $\bullet$ & \\
\hline Italy & $\bullet \bullet \bullet$ & $\bullet \bullet$ & $\bullet \bullet$ & & & & & & $\cdots \bullet$ & & $\bullet$ & \\
\hline Latvia & $\bullet$ & & $\bullet$ & & & $\bullet$ & & & $\bullet$ & & & $\bullet$ \\
\hline Lithuania & $\bullet \bullet \bullet \bullet$ & $\bullet$ & $\bullet$ & $\bullet$ & & $\bullet$ & $\bullet$ & & $\bullet$ & & $\bullet$ & \\
\hline Luxembourg & $\bullet \bullet$ & $\bullet \bullet$ & $\bullet$ & $\bullet \bullet$ & & & & & & $\bullet \bullet \bullet$ & $\bullet$ & \\
\hline Malta & $\bullet \bullet \bullet$ & $\bullet$ & $\bullet$ & $\bullet$ & & & & & $\bullet$ & & $\bullet$ & \\
\hline Netherlands & $\bullet$ & $\bullet$ & $\bullet \bullet \bullet$ & $\bullet$ & & $\bullet$ & $\bullet$ & & & $\bullet$ & & \\
\hline Poland & 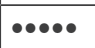 & 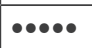 & $\bullet \bullet$ & $\bullet \bullet$ & & 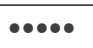 & $\bullet$ & & $\bullet \bullet \bullet \bullet$ & 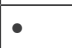 & $\bullet \bullet$ & \\
\hline Portugal & $\bullet$ & & $\bullet \bullet \bullet$ & $\bullet$ & & $\bullet$ & $\bullet$ & $\bullet$ & $\bullet$ & & $\bullet$ & \\
\hline Romania & $\bullet \bullet \bullet$ & $\bullet$ & $\bullet \bullet \bullet$ & & & $\bullet$ & & & $\bullet \bullet \bullet \bullet$ & & $\bullet$ & \\
\hline Slovakia & $\bullet$ & $\bullet$ & $\bullet$ & & & $\bullet$ & & & $\bullet$ & $\bullet \bullet$ & $\bullet$ & \\
\hline Slovenia & $\bullet$ & $\bullet \bullet$ & $\bullet \bullet \bullet$ & & & & & & $\bullet \bullet \bullet$ & $\bullet$ & & $\bullet$ \\
\hline Spain & $\bullet \bullet$ & $\bullet$ & $\bullet \bullet \bullet$ & $\bullet \bullet$ & $\bullet$ & & & & $\bullet \bullet$ & & $\bullet$ & \\
\hline Sweden & $\bullet \bullet \bullet$ & & $\bullet \bullet \bullet$ & & & & & $\bullet$ & $\bullet$ & & $\bullet$ & $\bullet$ \\
\hline United Kingdom & 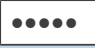 & $\bullet \bullet \bullet$ & 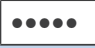 & $\bullet$ & $\bullet$ & $\bullet$ & & & $\bullet \bullet \bullet$ & & $\bullet$ & \\
\hline \multicolumn{13}{|l|}{ European Economic Area } \\
\hline Iceland & $\bullet \bullet$ & $\bullet$ & $\bullet \bullet$ & & & & & & & & & $\bullet$ \\
\hline Norway & $\bullet \bullet$ & $\bullet \bullet \bullet$ & $\bullet$ & $\bullet$ & & & & & $\bullet$ & & $\bullet$ & \\
\hline \multicolumn{13}{|l|}{ Other countries } \\
\hline Albania & $\bullet$ & $\bullet$ & $\bullet$ & $\bullet$ & & - & & & $\bullet$ & & & $\bullet$ \\
\hline Bosnia and Herzegovina & $\bullet$ & & $\bullet$ & $\bullet$ & & & & $\bullet$ & $\bullet$ & & & \\
\hline $\begin{array}{l}\text { Former Yugoslav Republic } \\
\text { of Macedonia }\end{array}$ & $\bullet \bullet \bullet \bullet$ & $\bullet$ & $\bullet \bullet \bullet \bullet$ & $\bullet$ & & $\bullet \bullet \bullet \bullet$ & & & $\bullet \bullet \bullet$ & $\bullet$ & $\bullet$ & • \\
\hline Kosovo* & $\bullet \bullet \bullet$ & & $\bullet$ & & & & & $\bullet$ & $\bullet \bullet \bullet$ & & & $\bullet$ \\
\hline Montenegro & $\bullet$ & & $\bullet$ & & & & & & $\bullet \bullet$ & & - & $\bullet$ \\
\hline Serbia & $\bullet$ & & $\bullet$ & & & & & & $\bullet$ & & & $\bullet$ \\
\hline Turkey & 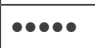 & $\bullet \bullet$ & $\bullet \bullet \bullet$ & $\bullet$ & & & & & $\bullet \bullet \bullet$ & $\bullet \bullet \bullet$ & & $\bullet$ \\
\hline
\end{tabular}

Each dot corresponds to one activity-year.

* This designation is without prejudice to positions on status, and is in line with United Nations Security Council Resolution 1244/99 and the International Court of Justice Opinion on the Kosovo declaration of independence. 
available. As a result, year on year, in their reply to the annual questionnaire, countries have expressed their strong support for ECDC to continue its work on the EAAD. This has also been highlighted by a steadily increasing number of countries participating and the growing interest of varied professional and stakeholder organisations.

Evaluation of EAAD in terms of understanding its impact on antibiotic consumption and on antibiotic resistance is difficult because (i) the effects will vary depending on the country as a result of variations in the extent and the intensity of the national campaign in each country and (ii) these effects are unlikely to be immediate as shown from previous national campaigns in some Member States. In addition, it is important to remember that since the campaigns have been applied heterogeneously at national levels, according to local needs and resources, a one size fits all impact analysis evaluation is not appropriate.

Regular opinion polls, i.e. 'Special Eurobarometers' on antimicrobial resistance commissioned by the European Commission, however, should help identify improvements in the knowledge, perception and selfreported attitudes of Europeans with antibiotics $[17,18]$. Additionally, the effects on antibiotic consumption and on antibiotic resistance in the European countries most active in the campaigns should become visible in the data reported to the European Surveillance of Antimicrobial Consumption Network (ESAC-Net) and the European Antimicrobial Resistance Surveillance Network (EARS-Net), respectively [19-21]. By providing training support on the development, implementation and evaluation of prudent antibiotic use campaigns, ECDC hopes that common evaluation indicators can now be developed at European level and implemented as part of national campaigns from 2014 onwards.

In 2013, ECDC and its partners launched the 6th edition of EAAD on 15 November 2013, with the emphasis that 'Everyone is responsible' for addressing antibiotic resistance and for using antibiotics more prudently, during a European Commission press conference [22]. During this press conference, the results of a recent 'Special Eurobarometer' on the attitudes and knowledge of Europeans about antibiotics, were presented together with a review of new research initiatives related to antimicrobial resistance and the latest surveillance data on resistance trends [21,23]. In particular, the 'Special Eurobarometer' on antimicrobial resistance showed a 5\% decrease between 2009 and 2013, in the percentage of Europeans who took antibiotics during the past year and an increasing awareness of Europeans that antibiotics do not kill viruses [18]. These are positive developments that may reflect the continuous efforts made by Member States in the framework of EAAD. This is also the rationale for an annual EAAD to support to national campaigns.

In 2013, ECDC arranged for a first extended global Twitter conversation with its partners in the United
States (US), Canada and Australia, and in connection with an EAAD Twitter chat organised jointly with the European Commission and WHO/Europe using the hashtag \#EAAD. Dedicated EAAD Twitter (@EAAD_EU) and Facebook (http://facebook.com/eaad.eu) accounts have been set up for the first time. The full evaluation of the 2013 edition of EAAD is currently ongoing. In reply to the annual questionnaire sent by ECDC to evaluate the activities in 2013, 22 of 41 responding countries highlighted that there was a change in their country that could be attributed to the momentum created by EAAD.

Looking to the future, self-medication with antibiotics has been identified as a new focus for EAAD 2014. Concerns about antimicrobial resistance and the need for a more prudent use of antibiotics are of global significance and are progressively being raised on political agendas. A growing number of countries and regions across globe, including the US, Canada and Australia, have aligned the timing of their activities to that of EAAD and the week of 18 November is increasingly being recognised as the moment to raise awareness about prudent use of antibiotics. This is a strong encouragement for the coordinators of the EAAD to continue acting as a global catalyst for discussion and raising awareness about prudent use of antibiotics.

Members of the European Antibiotic Awareness Day Technical Advisory Committee (i.e., experts and stakeholders who provided technical advice to ECDC as part of the Technical Advisory Committee during 2007-2012):

R. Muchl (Austria)a , J. Eyckmans (Belgium), J. Šturma (Czech Republic), H. Žemličkováa (Czech Republic), V Jindráka (Czech Republic); E. Parviainen (Finland), B. Schlemmer ${ }^{\text {(France), }}$ A. Lepape (France), M. Valtier (France), H. Giamarellou ${ }^{a}$ (Greece), A. Antoniadou (Greece), G. Daikos (Greece), M. Melles $^{a}$ (Hungary), M. Grazia Pompa (Italy); P. Casolari (Italy), S. Caplinskas (Lithuania), M. Borga (Malta), I.C. Gyssens $^{\text {a }}$ (The Netherlands), T. Verheij (The Netherlands), H.P. Muñiz ${ }^{a}$ (Norway), H. Nøkleby ${ }^{a}$ (Norway), W. Hryniewicz ${ }^{a}$ (Poland), M. Szjakowska (Poland), P. Ribeiro Da Silva (Portugal), S. Kovacsova (Slovakia), M. Vrdelja ${ }^{a}$ (Slovenia), J. Campos ${ }^{\text {a }}$ (Spain), C. Llor (Spain), O. Cars ${ }^{\text {a }}$ (Sweden), A.-L. How (Sweden), A. Sten (Sweden), K. Wahlberga (Sweden), E. Gilgunn-Jones (United Kingdom), D. Lecky (United Kingdom \& e-Bug), C. McNulty (United Kingdom \& e-Bug), C. Butler (UK), N. Heine/N. Safrany/A. Walters/V. Houdry/B. Toussaint/A. Gijsens/M. Kokki (DG SANCO, European Commission), A. Van Hengel/J. Bunikis/A. Lönnroth Sjödén (DG RESEARCH, European Commission), D. Lo Fo Wong/K. de Joncheere/H. Kruse/V. Hafner/ B. Ganter/A.P. Coutinho/R. Andraghetti/O. Polishchuk/H. Kluge/N. Emiroglu/H. Bak Pedersen (WHO/ Europe), R. Norrby (ESCMID), B. Beger/L. Tiddens-Engwirda (CPME), J. Chave (PGEU), G.Ferreira (EPSA).

Members of the European Antibiotic Awareness Day Collaborative Group who contributed at least one year during 2008-2012:

Austria: R. Strauss, P. Apfalter, S. Metz-Gercek, H. Mittermayer ${ }^{\mathrm{b}}$; Belgium: S. Coenen; Bulgaria: T. Kantardjiev, T. Velinov, B. Todorova; Croatia: A. Tambić Andrašević; Cyprus: N. Paphitou, C. Hadjianastasiou, M. Alexandrou, D. 
Pieridou-Bagatzouni; Denmark: A.M. Hammerum, R. Skov, K. Fuursted, G. Strøbæk, N. Frimodt-Møller; Estonia: K. Kutsar, M. Muzotsin; Finland: J. Jalava, A. Hakanen, M. Gunell, O. Lyytikäinen, J.Vuopio, P. Huovinen; France: J.-M. Azanowsky; Germany: A. Ziegelmann, K. de With; Hungary: K. Böröcz, A. Kurcz, I. Luif, E. Szilágyi;

Iceland: T. R. Thorsteinsdottir, T. Gudnason, G. Sigmundsdottir, J. Hedinsdottir, K. Kristinsson, H. Briem; Ireland: R. Cunney; Italy: A. Pantosti, P. Salcuni; Latvia: U. Dumpis, S. Terela; Lithuania: A. Sinkeviciute, R. Valinteliene; Luxembourg: S. Christmann, E. Heisbourg;

Malta: P. Zarb; The Netherlands: S. de Greeff, J. Prins, L. Wijgergangs; Norway: M. Steinbakk, G. Wøien, M. Lindbæk, G.S. Simonsen; Poland: B. Mazinska, A. Olczak Pieńkowska; Portugal: J.A. Paiva, A.C. Costa, J. Melo Cristino; Romania: I. Codita, A. Băicuș, A. Canton; Slovakia: L. Siegfried, H. Hupková; Slovenia: M. Čižman, A. Svetlin, S. Rojs; J. Kolman; Spain: J. Oteo; B. Aracil; M. Pérez-Vázquez; Sweden: I. Riesenfeld-Örn, J. Struwes, A. Tegnell; United Kingdom: S. Wellsteed, M. Robinson, L. Willock; D. Ashiru-Oredope; Montenegro: G. Mijović; The Former Yugoslav Republic of Macedonia: G. Bosevska; Serbia: Z. Jelesić; Turkey: N. Çöplü; H. Şimşek; Albania: P. Pipero, A. Ylli; Bosnia and Herzegovina: M. Hukić; Kosovo*: L. Raka.

a Members of both the European Antibiotic Awareness Day Technical Advisory Committee and of the European Antibiotic Awareness Day Collaborative Group.

b Deceased.

* This designation is without prejudice to positions on status, and is in line with United Nations Security Council Resolution $1244 / 99$ and the International Court of Justice Opinion on the Kosovo declaration of independence.

\section{Conflict of interest}

None declared.

\section{Authors' contributions}

Sarah Earnshaw - lead author, drafted article; Giovanni Mancarella - co-lead author, drafted article; Andrea Mendez - evaluation; Boyana Todorova - EAAD website; Marybelle Stryk - EAAD social media; Anna-Pelagia Magiorakos - EAAD patient stories and introduction; Enrico Possenti - EAAD audiovisuals; Signe Gilbro - EAAD multi-lingual content; Herman Goossens - EAAD Technical Advisory Committee; Barbara Albiger - Figures, references and revision article; Dominique Monnet - discussion and list of acknowledgements.

\section{References}

1. Cosgrove SE. The relationship between antimicrobial resistance and patient outcomes: mortality, length of hospital stay, and health care costs. Clin Infect Dis. 2006;42 Supp 2:S82-9. http://dx.doi.org/10.1086/499406

2. Carlet J, Jarlier V, Harbarth S, Voss A, Goossens H, Pittet D, et al. Ready for a world without antibiotics? The Pensières Antibiotic Resistance Call to Action. Antimicrob Resist Infect Control. 2012;1(1):11. http://dx.doi.org/10.1186/2047-2994-1-11

3. Council of the European Union. Council Recommendation of 15 November 2001 on the prudent use of antimicrobial agents in human medicine (2002/77/EC). Official Journal of the European Communities. 2002;45:13-6. Available from: http://eur-lex. europa.eu/LexUriServ/LexUriServ.do?uri=0J:L:2002:034:0013 :0016:EN:PDF

4. McNulty CA, Johnson AP. The European Antibiotic Awareness Day. J Antimicrob Chemother. 2008;62(5):853-4. http://dx.doi. org/10.1093/jac/dkn410

5. Spyridis N, Sharland M. The European Union Antibiotic Awareness Day: the paediatric perspective. Arch Dis
Child. 2008;93(11):909-10. http://dx.doi.org/10.1136/ adc.2008.149625

6. Earnshaw S, Monnet DL, Duncan B, O’Toole J, Ekdahl K, Goossens H, et al. European Antibiotic Awareness Day, 2008 the first Europe-wide public information campaign on prudent antibiotic use: methods and survey of activities in participating countries. Euro Surveill. 2009;14(30)=19280.

7. European Centre for Disease Prevention and Control (ECDC). European Antibiotic Awareness Day. Stockholm: ECDC; Available from: http://www.ecdc.europa.eu/en/eaad/Pages/ toolkits.aspx).

8. Eurosurveillance editorial team. European Antibiotic Awareness Day provides platform for campaigns on prudent use of antibiotics for the fourth time. Euro Surveill. 2011;16(46). pii= 20018.

9. Eurosurveillance editorial team. Fifth European Antibiotic Awareness Day on 18 November: joining forces to reduce antibiotic resistance. Euro Surveill. 2012;17(46). pii= 20314 .

10. Codiță I. Participation of Romania in the ECDC (European Center for Disease Control) initiative of the Antimicrobial Resistance Awareness Day 2009. Bacteriol Virusol Parazitol Epidemiol. 2010;55(2):74-6, 71-3. English, Romanian.

11. Mazinska B, Hryniewicz W. Kampania edukacyjna Europejski Dzien Wiedzy o Antybiotykach--czy wplynela na zmiane postaw spoleczenstwa w Polsce? [European Antibiotic Awareness Day educational campaign--has it changed public attitudes to antibiotic use in Poland?]. Pol Merkuriusz Lek. 2010;29(173):296-303. Polish.

12. Leaper D. European Union Antibiotic Awareness Day relevance for wound care practitioners. Int Wound J. 2010;7(5):314-5. http://dx.doi.org/10.1111/j.1742-481X.2010.00740.x

13. Finch R, Sharland M. 18 November and beyond: observations on the EU Antibiotic Awareness Day. J Antimicrob Chemother. 2009;63(4):633-5. http://dx.doi.org/10.1093/jac/dkpo39

14. Hryniewicz W, Mazinska B. Europejski dzien wiedzy o antybiotykach--dlaczego potrzebny? [European Antibiotic Awareness Day--why needed?]. Pol Merkuriusz Lek. 2009;27(160):261-4. Polish.

15. Lewis MA. Why we must reduce dental prescription of antibiotics: European Union Antibiotic Awareness Day. Br Dent J. $2008 ; 22 ; 205(10): 537-8$

16. Stockley JM. European Antibiotic Awareness Day 2010: why doesn't promoting antibiotic awareness always work? J Infect. 2010;61(5):361-3. http://dx.doi.org/10.1016/j.jinf.2010.09.003

17. Special Eurobarometer 338. Antimicrobial resistance, November-December 2009. Brussels, TNS Opinion \& Social 2010. Available from: http://ec.europa.eu/health/ antimicrobial resistance/docs/ebs 338 en.pdf

18. Special Eurobarometer 407. Antimicrobial resistance, November 2013. Brussels, TNS Opinion \& Social 2010. Available from: http://ec.europa.eu/health/ antimicrobial_resistance/docs/ebs_407_sum_en.pdf

19. European Centre for Disease Prevention and Control (ECDC). Surveillance of antimicrobial consumption in Europe, 2011. Stockholm: ECDC; 2014. Available from: http://ecdc.europa. eu/en/publications/Publications/antimicrobial-consumptioneurope-surveillance-2011.pdf

20. European Centre for Disease Prevention and Control (ECDC). Antimicrobial resistance surveillance in Europe 2011. Annual report of the European Antimicrobial Resistance Surveillance Network (EARS-Net). Stockholm: ECDC; 2012. Available from: http://ecdc.europa.eu/en/publications/Publications/ antimicrobial-resistance-surveillance-europe-2011.pdf

21. European Centre for Disease Prevention and Control (ECDC). Antimicrobial resistance surveillance in Europe 2012. Annual report of the European Antimicrobial Resistance Surveillance Network (EARS-Net). Stockholm: ECDC; 2013. Available from: http://ecdc.europa.eu/en/publications/Publications/ antimicrobial-resistance-surveillance-europe-2012.pdf

22. European Commission. Press release database. European Antibiotic Awareness Day 2013: EU steps up its fight against drug-resistant bacteria. IP/13/1081 15/11/2013. Available from http://europa.eu/rapid/press-release_IP-13-1081_en.htm

23. European Centre for Disease Prevention and Control (ECDC). Carbapenemase-producing bacteria in Europe: interim results from the European Survey on carbapenemase-producing Enterobacteriaceae (EuSCAPE) project. Stockholm: ECDC; 2013. Available from: http://www.ecdc.europa.eu/en/publications/ Publications/antimicrobial-resistance-carbapenemaseproducing-bacteria-europe.pdf 